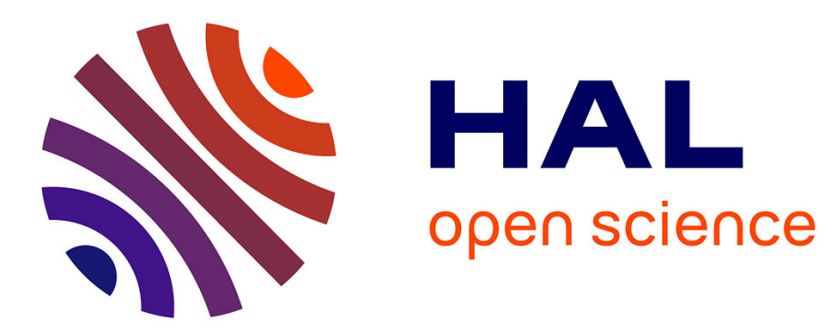

\title{
Monte Carlo Simulations of Low Energy Electrons in Silicon
}

\author{
J. Pierron, C. Inguimbert, M. Belhaj, M. Raine, J. Puech
}

\section{To cite this version:}

J. Pierron, C. Inguimbert, M. Belhaj, M. Raine, J. Puech. Monte Carlo Simulations of Low Energy Electrons in Silicon. RADECS 2016, Sep 2016, BREME, Germany. hal-01442097

\section{HAL Id: hal-01442097 \\ https://hal.science/hal-01442097}

Submitted on 20 Jan 2017

HAL is a multi-disciplinary open access archive for the deposit and dissemination of scientific research documents, whether they are published or not. The documents may come from teaching and research institutions in France or abroad, or from public or private research centers.
L'archive ouverte pluridisciplinaire HAL, est destinée au dépôt et à la diffusion de documents scientifiques de niveau recherche, publiés ou non, émanant des établissements d'enseignement et de recherche français ou étrangers, des laboratoires publics ou privés. 


\title{
Monte Carlo Simulations of Low Energy Electrons in Silicon
}

\author{
J. Pierron, C. Inguimbert, M. Belhaj, M. Raine and J. Puech
}

\begin{abstract}
The electron transport in silicon at low and very low energy $(10 \mathrm{eV}-2 \mathrm{keV})$ is investigated with a Monte Carlo code. The elastic scattering with nuclei is described by Mott's model of partial waves, whereas the inelastic collisions with electrons are described by the complex dielectric function theory. The code has been validated by means of comparison with electron emission yields (EEY) and energy loss spectra experimentally measured in ultrahigh vacuum on an Ar-etched sample. Electron emission yields, practical ranges, and ionizing doses are presented for electrons in silicon down to $10 \mathrm{eV}$.
\end{abstract}

Index terms - Electron emission yield, low energy electrons, Monte Carlo code, practical range.

\section{INTRODUCTION}

$\mathrm{T}$ he study of low energy electron transport in matter is of great interest for many fields of applications. For instance, in microelectronics, the shrinking of electronic devices has recently reached about $14 \mathrm{~nm} \mathrm{[1]} \mathrm{and} \mathrm{thus} \mathrm{has}$ created a need for more precise electron transport codes at very low energy to study the effects of ion and electron trackstructures on the sensitivity of electronic devices [2] [3]. At these scales, the radiation transport modeling shall be accurate over small distances of few nanometers and very low energies of few tens of $\mathrm{eV}$. Some authors have worked to develop numerical methods adapted to the electron transport at low energy [2] [3] [4] [5] [6] [7] [8]. Recently, a new module dedicated to low energy electron transport has been implemented in Geant4. This electromagnetic model of Geant4 called MicroElec performs the electrons transport down to $\sim 16 \mathrm{eV}$ [4] [5] [6]. It has been used to improve the modeling of heavy ion track structures below a radial distance of $10 \mathrm{~nm}$ [4]. The Geant4-MicroElec library extension [3] [4] [5] [6] has also been employed to investigate the sensitivity of advanced devices to incident electrons [9]. For now, the Geant4MicroElec model is only valid down to $\sim 16 \mathrm{eV}$ for a single material (silicon). Some other codes have been implemented over the past years for silicon [2] [7]. ONERA in collaboration with CNES has developed its own code: OSMOSEE [10] [11]. In this code dedicated to low energy electron emission, the implemented physical models were initially specific to aluminum. On the other side, the Geant4-MicroElec library extension is based on the complex dielectric function theory

This paper was submitted to RADECS in September 2016.

J. Pierron, C. Inguimbert and M. Belhaj are with ONERA, 2 av. E. Belin, 31055 Toulouse, France (tel: 33-562252734, email: Juliette.Pierron@onera.fr, Christophe.Inguimbert@onera.fr, Mohamed.Belhaj@onera.fr). M. Raine is with CEA, DAM, DIF, F-91297 Arpajon, France (email: Melanie.Raine@cea.fr ). J. Puech is with CNES, 18 av. E. Belin, 31401 Toulouse, France (email: Jerome.Puech@cnes.fr). and could be easily adapted to different materials. In a collaborative effort, this work proposes to extend the domain of validity of such kind of approaches to different materials and down to a few eV. At these low energies additional phenomena (not included in MicroElec), for example the crossing of the surface potential barrier or surface plasmon excitations, must be taken into account. Up to now, three materials (aluminum, silver and silicon) have been implemented in the code OSMOSEE for energies between $10 \mathrm{eV}$ and $2 \mathrm{keV}$ [12]. This enables to estimate some relevant parameters for new generation devices of small dimensions such as the range of electrons and the ionizing dose.

The validation of the model is performed by means of comparisons with experimental measurements obtained with a facility entirely dedicated and designed to electron emission [10] [13]. Electron Emission Yield (EEY) and energy loss spectra obtained in ultrahigh vacuum on an Ar-etched sample of silicon are compared with simulation results.

\section{LOW ENERGY MONTE CARLO CODE}

If electron transport in matter is well described at high energies, it is not the case at low (few tens of eV) and very low energy (a few eV) where most analytical models extrapolated from high energy fail [14]. In matter the trajectory of the electrons is driven by two main mechanisms: the elastic and the inelastic scatterings. At low energy, one has to take into account not only the inelastic scattering (i.e. collision with electrons), but also the elastic scattering (i.e. deflection by nuclei) that is predominant in these energy ranges. In the code OSMOSEE, the elastic interactions are described by Mott's model (or partial waves) from the code ELSEPA [15]. The inelastic scattering is described by the complex dielectric function theory and optical data [3] [16]. The code takes into account surface plasmon excitations and the crossing of the surface potential barrier [10] [11]. The MC procedure is a standard one. Random numbers are used to determine the type of interaction, the distance traveled between collisions, and the characteristics of the interaction (scattering angles, energy transfers). It has been described in previous papers [10] [11]. Paths and energy losses are calculated until the electron is emitted out of the solid or until its energy falls under an energy threshold. This threshold equals $e \chi+E_{G}+\Delta V$ for semiconductors, with $E_{G}$ the energy bandgap, e $\chi$ the electron affinity, and $\Delta \mathrm{V}$ the valence bandwidth.

First, the principle of the simulation method (complex dielectric function theory) is briefly described in section III. Next, simulation results (energy spectra, EEY, practical ranges, and 
ionizing doses) performed with the code OSMOSEE in silicon targets are presented in sections IV, V, and VI.

\section{INELASTIC MEAN FREE PATHS}

In the complex dielectric function theory, the total inelastic cross section $\sigma$ can be obtained using equation (1) where $N Z$ is the electronic density of the solid, $\mathrm{a}_{0}=0.529 \AA$ the Bohr radius, $T$ the kinetic energy of the incident electron, and $\varepsilon(\hbar \omega, \hbar q)$ the exact dielectric function of the solid with $\hbar \omega$ and $\hbar q$ the energy and momentum transferred to the electrons of the solid [2] [3].

$$
\frac{d^{2} \sigma}{d \hbar \omega d \hbar q}=\frac{1}{N Z} \frac{1}{\pi a_{0} T} \Im m\left[\frac{-1}{\varepsilon(\hbar \omega, \hbar q)}\right] \frac{1}{\hbar q}
$$

The first step of this method is to model the imaginary part of the inverse of the dielectric function (energy loss function) that contains all information on the electronic band structure of the solid. The energy loss function for $\hbar q=0$ may be obtained from optical data [16]. Methods to extend this function to all momentums $\hbar q$ have been described by different authors [2] [3] [8] [17] [18]. Consistency of the fitting is given by three sum-rules [3]. We found a maximum discrepancy of $8 \%$ for silicon.

To get the total inelastic mean free path, one has to integrate (1) over energy $\hbar \omega$ and momentum $\hbar q$ transfers. The boundaries in $\hbar q$ are determined [3] [17] by the law of conservation of energy and momentum (2).

$$
q_{\mp}(T, \hbar \omega)=\frac{\sqrt{2 m}}{\hbar}[\sqrt{T} \mp \sqrt{T-\hbar \omega}]
$$

The boundaries in energy transfer $\hbar \omega$ are obtained with respect to the Pauli principle [3] [8] [17]. In the MC code, the bottom of the valence band has been chosen as an energy reference for electrons in semiconductors. Equations (3) and (4) provide respectively the total inelastic mean free path $\lambda$ and the stopping power $S P, E$ being the total energy of the electron in the solid.

$$
\begin{gathered}
\lambda^{-1}(E)=\frac{1}{\pi a_{0}\left(E-E_{G}-\Delta V\right)} \times \\
\int_{E_{G}}^{E-E_{G}-\Delta V} \int_{q_{-}\left(E-E_{G}, \hbar \omega\right)}^{q_{+}\left(E-E_{G}, \hbar \omega\right)} \Im m\left[\frac{-1}{\varepsilon(\hbar \omega, \hbar q)}\right] \frac{1}{\hbar q} d \hbar q d \hbar \omega \\
S P(E)=\int_{E_{G}}^{\frac{E+E_{G}}{2}} \hbar \omega \frac{d \lambda^{-1}(\hbar \omega)}{d \hbar \omega} d \hbar \omega
\end{gathered}
$$

These calculations are presented on Fig. 1 and Fig. 2. As depicted on these figures, the calculated total inelastic mean free path and stopping power are in good agreement with data from the literature.

In the code OSMOSEE, the surface plasmon excitations are taken into account by replacing the energy loss function
$\mathfrak{I} m\left[\frac{-1}{\varepsilon(\hbar \omega, \hbar q)}\right]$ by $\mathfrak{I} m\left[\frac{-1}{\varepsilon(\hbar \omega, \hbar q)+1}\right]$ with $\varepsilon(\hbar \omega, \hbar q)+1=0$ the dispersion relation for collective excitations near the surface [17]. A dependence of the electron depth $\mathrm{z}$ (i.e. Begrenzung effect) is then added to the surface plasmon mean free path $\lambda_{s p}$ [8] [10] [11] with equation (5), $\lambda_{v p}$ being the volume plasmon mean free path, and $\hbar \omega_{s}$ being the surface plasmon energy.

$$
\begin{aligned}
& \lambda_{s p}^{-1}=\lambda_{v p}^{-1} \cdot\left[\exp \left(-\frac{z}{z_{0\left(\hbar \omega_{s}\right)}}\right)\right] \\
& \text { where } z_{0}(\hbar \omega) \approx 3.90 \frac{\sqrt{T}}{\hbar \omega}(\text { in } \AA)
\end{aligned}
$$

According to this model, the surface plasmon excitations may occur only in the first atomic layers from the surface [8]. As a result, the probability for exciting surface plasmons increases at very low energy.

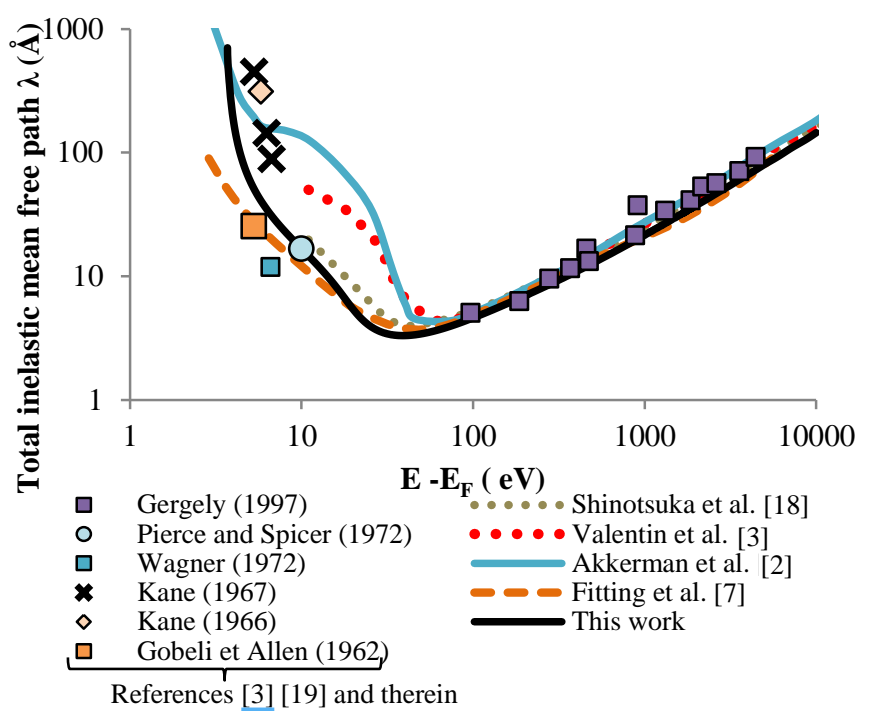

Fig. 1. Total inelastic mean free path for electrons in silicon. Full [2], dashed [7] and dotted [3] [18] lines represent calculations. Dots represent experimental data from references [3] [19] and therein, and crosses are used for calculations from [19] and therein.

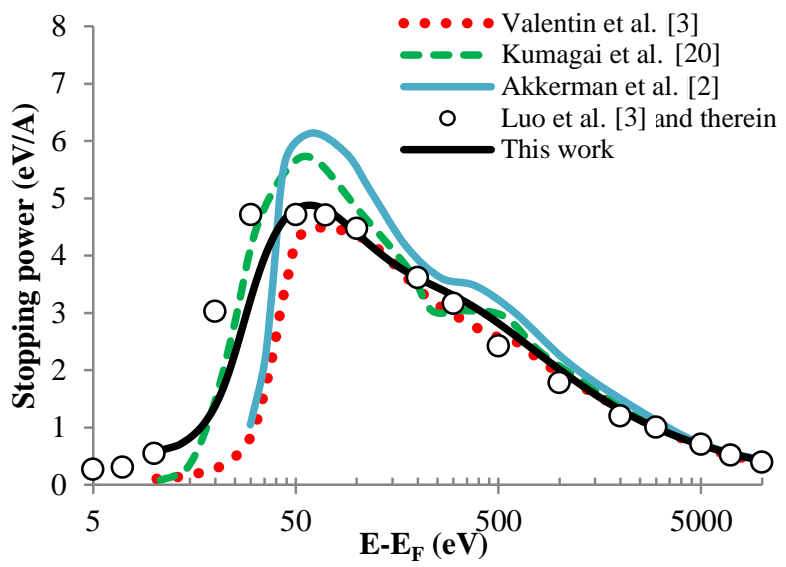

Fig. 2. Stopping power for electrons in silicon. Full [2], dashed [20] and dotted [3] lines represent calculations. Dots represent experimental data from [3] and therein. 


\section{VALIDATION: ENERGY LOSS SPECTRA AND EEY}

The code OSMOSEE has been validated by comparisons with the literature and experimental measurements of different physical quantities, such as the energy loss spectra and the Electron Emission Yield (EEY).

Some backscattered electrons are primary electrons that have undergone inelastic energy losses. The electrons that have been backscattered after experiencing a single inelastic interaction will get out of the target material with a typical energy loss $\hbar \omega$ representative of the interaction. On an energy loss spectrum, that leads to a typical structure at the characteristic energy $E=E_{0}-\hbar \omega$, with $E_{0}$ the incident electron beam energy. Usually for backscattered electrons, the spectra are given in function of the energy loss $\hbar \omega=E_{0}-E$.

The energy loss spectra measurements were performed in an ultrahigh vacuum facility on an Ar-etched sample of silicon. A full description of the experimental facility can be found in reference [13] and therein. Fig. 3 shows that a good agreement is found between MC simulations and experimental measurements. The peaks corresponding to the volume plasmon excitation (vp) and its multiples appear clearly at $\hbar \omega=16.8,33.6$ and $50.8 \mathrm{eV}$. The surface plasmon excitation (sp) corresponds to the shoulder at $11.6 \mathrm{eV}$. It is less noticeable than the volume plasmon excitations on both simulated and experimental spectra [21] (and therein).

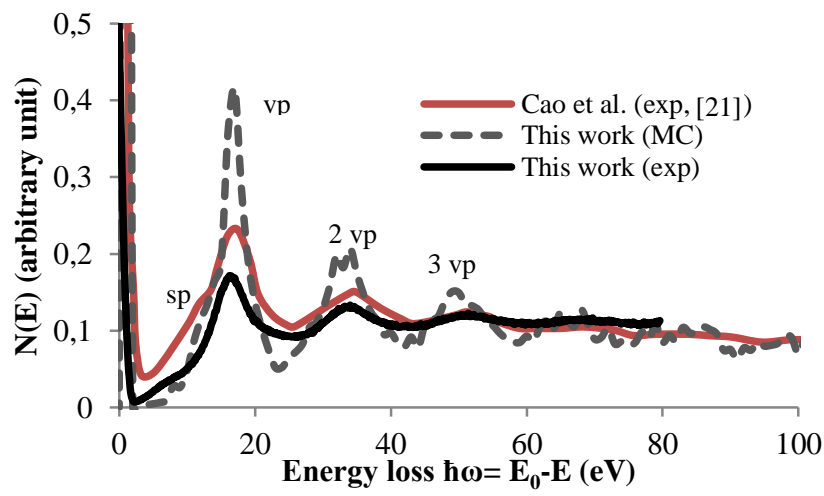

Fig. 3. Energy loss spectrum for backscattered electrons in silicon. Our experimental spectrum corresponds to $\mathrm{E}_{0}=1990 \mathrm{eV}$ whereas our MC spectrum (dashed line) and the experimental measurements of Cao et al. [21] are for $\mathrm{E}_{0}=1000 \mathrm{eV}$.

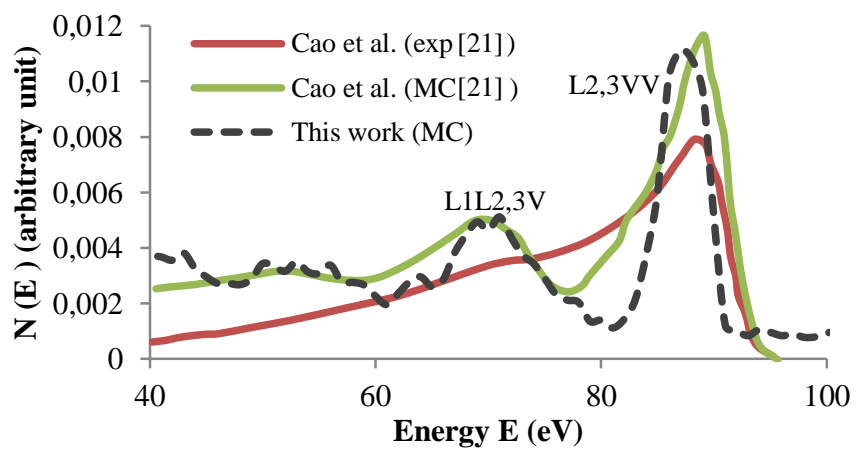

Fig. 4. Energy spectrum for the $\mathrm{L}_{2,3} \mathrm{VV}$ Auger transition in silicon. Full lines are $\mathrm{MC}$ simulation and experimental measurements of [21]. The dashed line is our MC simulation.
In addition to the backscattered electrons, the study of the Auger electrons is useful to validate a MC code. The Auger electrons are secondary electrons born from collisions with core electrons of the solid. They are emitted out of the solid with a typical energy that depends on the core levels involved in the interaction. Fig.4 shows the MC results for the $\mathrm{L}_{2,3} \mathrm{VV}$ and $\mathrm{L}_{1} \mathrm{~L}_{2,3} \mathrm{~V}$ Auger transition in silicon. Our MC simulations are in fairly good agreement with the MC simulations and the experimental results of Cao et al. [21].

The EEY is defined as the ratio between the number of electrons emitted by a solid over the number of electrons initially impacting this solid. For silicon, as depicted in Fig. 5, MC simulated yields are in good agreement with Bronstein \& Fraiman's experimental data [22], and with the MC simulations of Kuhr \& Fitting [8]. Since our measurements are performed on bulk solids, for easily oxidized materials such as silicon, a capping layer of oxide may remain on the sample after Ar-etching. This may explain the discrepancy at very low energy between our data and Bronstein and Fraiman's.

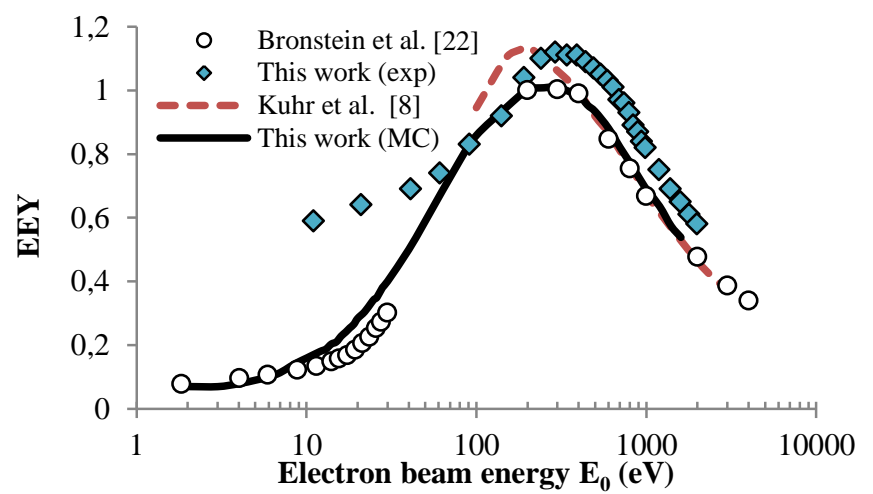

Fig. 5. Backscattered and secondary electron emission yields at low energy for silicon. Full lines are MC simulations [8], circles are Bronstein \& Fraiman's experimental data [22], and square are our experimental measurements.

\section{CSDA AND PRACTICAL RANGES}

In a solid, the incident electrons are slowed down by successive inelastic scattering with some electrons of the solid atoms. Each electron has an individual trajectory. The path length of this trajectory is the total distance traveled until the electron comes to rest. The average path length is known as the 'range' of the electrons in the solid [14] [23]. The range, in the Continuous-Slowing-Down Approximation (CSDA), can be estimated by integration of equation (4) over energy from a final to an initial value. Our simulations enable the estimation of the CSDA range down to a few eV as depicted in Fig.6.

However, due to deflections by nuclei, most electrons do not reach a depth that equals their range. The depth they finally reach is called the penetration depth. For electrons, it is common to define the average penetration depth, a metric more representative than the CSDA range of the penetration distance that can be traveled by the electrons in a solid. The practical range is commonly defined [14] (see Fig. 7) as the point where the tangent at the steepest section of the transmission curve intersects with the thickness or depth axis (X-axis). 


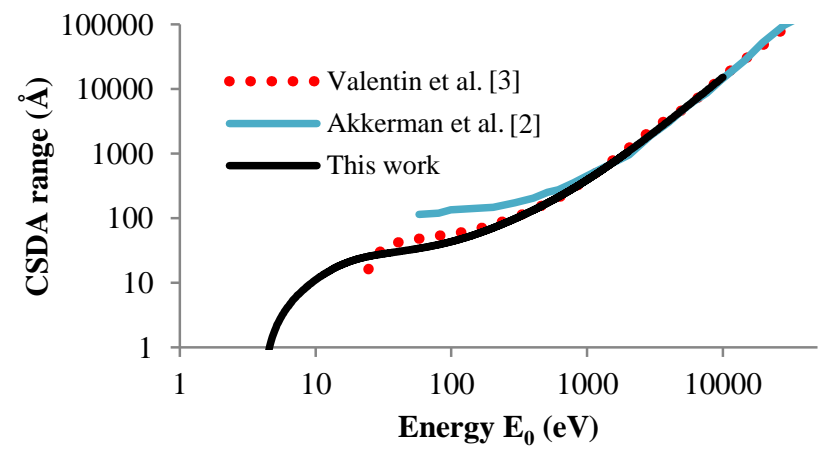

Fig. 6. CSDA ranges for electrons in silicon. The data are from [2] [3] and references therein.

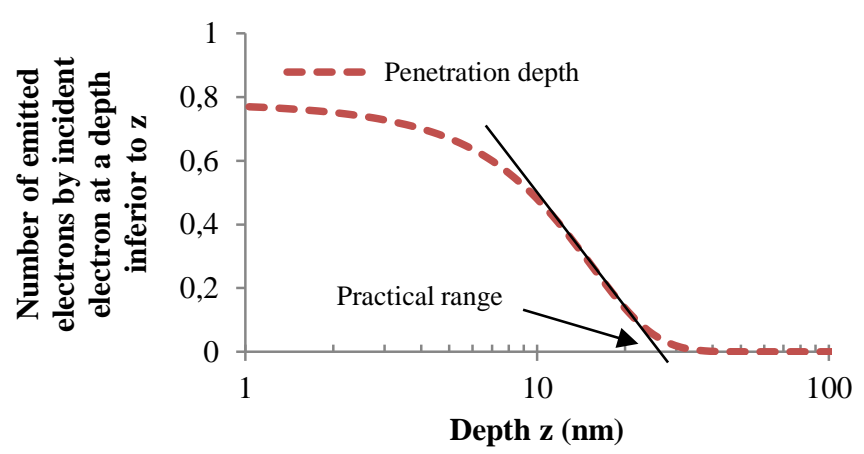

Fig. 7. Definition of the practical range for electrons. The curve is shown for electrons of $\mathrm{E}_{0}=800 \mathrm{eV}$ in silicon.

The practical range is plotted on Fig. 8. A good agreement is found with data from the literature at high energy, however as can be seen in Fig. 8, below $100 \mathrm{eV}$ the practical range tends to stabilize to a value of a few nanometers [23] instead of decreasing according to a power law as predicted by commonly used analytical models [14] [23].

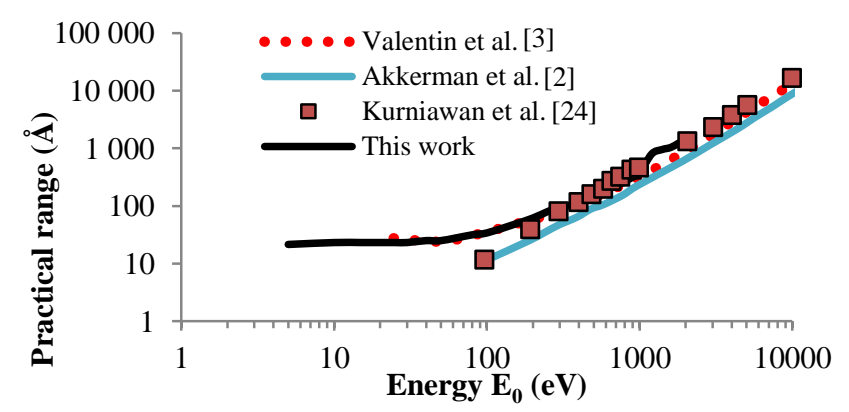

Fig.8. Practical ranges for electrons in silicon. The data come from [2] [3] and [24].

\section{IONIZING DOSE}

The ionizing dose is the deposited energy by unit of mass of a target material subsequent to interactions with the electrons of the solid. The code OSMOSEE enables the calculation of dose profiles down to a few eV. Fig. 9 shows the ionizing doses profiles for $100 \mathrm{eV}$ and $1 \mathrm{keV}$. As depicted in that figure, at $1 \mathrm{keV}$ our MC simulation are in fairly good agreement with the MC simulation of Akkerman et al. [2], and that of Valentin et al. [3]. At $100 \mathrm{eV}$, there is a huge discrepancy with the results of Valentin et al. who use the Geant4MicroElec library extension. This discrepancy may be explai- ned by the differences on the elastic and inelastic mean free paths, and on the energy cutoff at which the electrons come to rest in the solid.

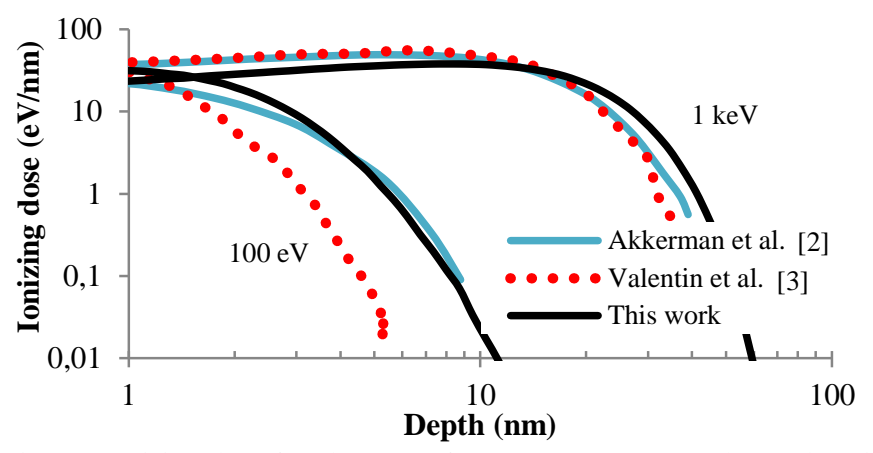

Fig. 9. Ionizing dose for electrons of energy $E_{0}=100 \mathrm{eV}$ and $E_{0}=1 \mathrm{keV}$ in silicon. The data come from [2] and [3].

The probability of the elastic scattering is shown in Fig. 10 for the three codes. For an incident electron, the number of elastic scatterings is the result of a competition between the elastic and the inelastic mechanisms. Fig. 10 shows that the probability of the elastic scattering presents a huge discrepancy at low energy. As previously depicted on Fig. 1, the inelastic mean free path of the code OSMOSEE is much lower below $50 \mathrm{eV}$ than those of Akkerman et al. and of Valentin et al. In the code OSMOSEE, the electrons tend to do more inelastic collisions at low energy. As a result, the electrons may go further in the solid and lose their energy faster. A discrepancy also exists for the elastic scattering. The code OSMOSEE uses the cross sections from the code ELSEPA of Salvat and coworkers [15] down to $10 \mathrm{eV}$. Valentin et al. employ the cross sections from the ICRU database [25] based on the code ELSEPA but above $50 \mathrm{eV}$. Below $50 \mathrm{eV}$, the Mott's model (or partial waves) has been extrapolated down to $10 \mathrm{eV}$ in the code OSMOSEE, whereas Akkerman et al. [2] and Valentin et $a l$. [3] employ the cross sections for $\mathrm{CS}_{2}$ from ab initio calculations by Bettega et al. [26].

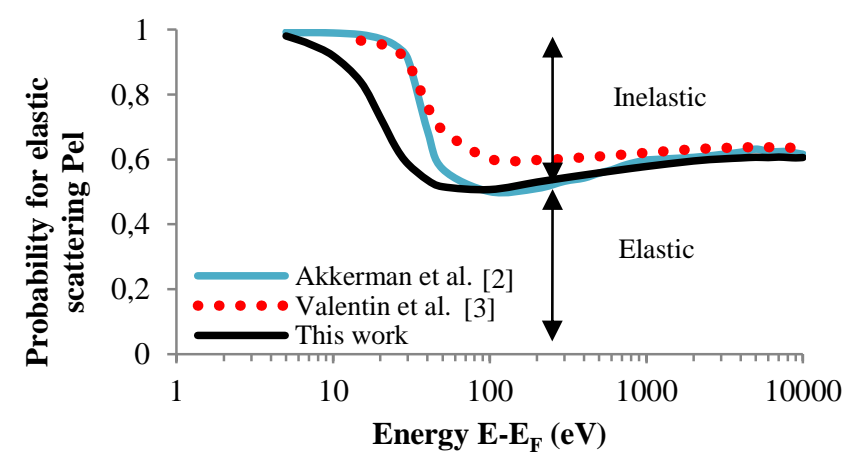

Fig. 10. Probability of elastic scattering calculated from the elastic and inelastic mean free paths for electrons in silicon in [2] [3] and in the code OSMOSEE.

In the code OSMOSEE, the energy cutoff is of $e \chi+E_{G}+$ $\Delta V$ with respect to the bottom of the valence band. This limit is equivalent to $e \chi=4.05 \mathrm{eV}$ in the vacuum. For the codes of Akkerman et al. and of Valentin et al., the energy cutoff is respectively of $5 \mathrm{eV}$ and of $16.7 \mathrm{eV}$. 
Fig. 11 presents the ionizing dose profiles calculated with the code OSMOSEE for electrons from 10 to $200 \mathrm{eV}$. Below $100 \mathrm{eV}$, as the electrons go slightly further into the solid, they mainly deposit their energy in the first few nanometers from the surface. As a result, the ionizing dose level increases near the surface. This behavior is in agreement with the practical range of electrons that shows a threshold below $100 \mathrm{eV}$ (Fig. 8). At $200 \mathrm{eV}$, as the electrons go deeper in the solid, they deposit their energy further into the solid. As a result, the maximum level of the ionizing dose is located deeper. This behavior is also shown on Fig. 9

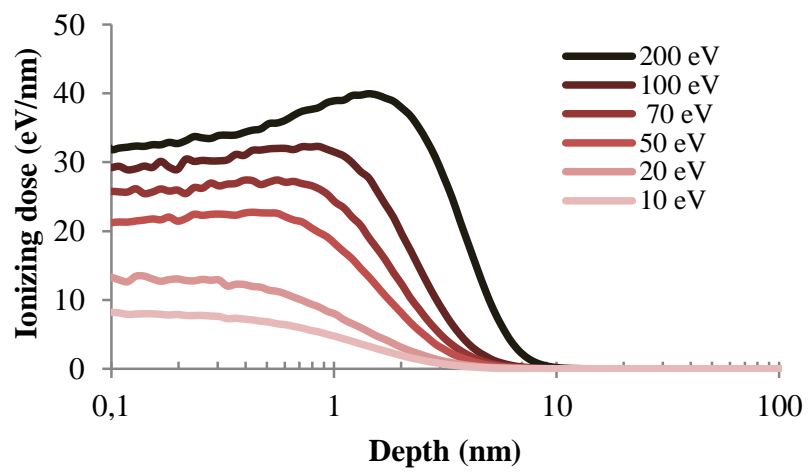

Fig. 11. Ionizing dose profiles for electrons of energy $\mathrm{E}_{0}=10,20,50,70,100$ and $200 \mathrm{eV}$.

\section{CONCLUSION}

The transport of electrons in silicon is investigated with a Monte Carlo code down to $10 \mathrm{eV}$. The MC code is based on the complex dielectric function theory and takes into account surface plasmon excitations. The code has been validated by comparisons with experimental measurements of electron energy loss spectra and electron emission yields performed in ultrahigh vacuum on an Ar-etched sample. Simulation results of practical ranges and ionization doses are in good agreement with the literature. The calculations of the ionizing dose profiles are given down to $10 \mathrm{eV}$. At low energy, the ionizing dose profiles show a correlation with the practical range. Below $100 \mathrm{eV}$, while the practical range of electrons stabilizes to a value of a few nanometers, the ionizing dose level increases near the surface. When increasing the energy of incidence, the electrons go further into the solid and the maximum ionizing dose level is located deeper.

\section{REFERENCES}

[1] G. Larrieu and X. L. Han, "Vertical nanowire array-based field effect transistors for ultimate scaling”, Nanoscale, vol. 5, nº, pp. 2437-2441, Mar. 2013.

[2] A. Akkerman, M. Murat and J. Barak, "Monte Carlo calculations of electron transport in silicon and related effects for energies of 0.02-200 keV", J. Appl. Phys., vol. 106, $\mathrm{n}^{\circ} 11$, pp. 113703-12, 2009.

[3] A. Valentin, M. Raine, J.-E. Sauvestre, M. Gaillardin, and P. Paillet, "Geant4 physics processes for microdosimetry simulation: Very low energy electromagnetic models for electrons in silicon", Nucl. Instrum.Meth. Phys. B., vol. 288, pp. 66-73, Oct 2012.

[4] M. Raine, A. Valentin, M. Gaillardin, and P. Paillet, "Improved simulation of ion track structures using new Geant 4 models - Impact on the modeling of advanced technologies response", IEEE Trans. Nucl. Sci., vol. 59, n6, pp. 2697-2703, Dec. 2012.

[5] M. Raine, M. Gaillardin and P. Paillet, "Geant4 physics processes for silicon microdosimetry simulation: Improvements and extension of the energyrange validity up to $10 \mathrm{GeV} /$ nucleon", Nucl. Instrum. Meth. B, vol. 325, pp. 97-100, Apr. 2014.

[6] Geant4 [Online]. Available at: https://geant4.web.cern.ch/geant4/

[7] H.-J. Fitting, E. Schreiber, J.-C.H. Kuhr, and A. Von Czarnowski, "Attenuation and escape depths of low-energy electron emission", J. Electron Spectrosc., vol. $119, \mathrm{n}^{\circ} 1$, pp. 35-47, Jul. 2001.

[8] J.-C. H. Kuhr and H.-J. Fitting, "Monte Carlo simulation of electron emission from solids", J. Electron Spectrosc., vol. 105, n²-3, pp. 257-273, Dec. 1999.

[9] C. Inguimbert, R. Ecoffet, and D. Falguère, "Electron Induced SEUs: Microdosimetry in Nanometric Volumes", IEEE Trans. Nucl. Sci., vol. 62, ${ }^{\circ}$ 6, pp. 2846-2852, Dec. 2015.

[10] J. Roupie, "Contribution à l'étude de l'émission électronique sous impact d'électrons de basse énergie ( $<=1 \mathrm{keV})$ : application à l'aluminium", $\mathrm{Ph} . \mathrm{D}$. dissertation, Université de Toulouse, France, 2013.

[11] J. Roupie, O. Jbara, T. Tondu, M. Belhaj and J. Puech, "The study of electron emission from aluminium in the very low primary energy range using Monte Carlo simulations", J. Phys. D. Appl. Phys., vol. 46, n¹2, pp. 125306, Feb. 2013.

[12] J. Pierron, C. Inguimbert, M. Belhaj and T. Gineste, "Monte-Carlo lowenergy electron transport in metals for secondary emission applications", presented at LEE2015 Conference, Hernstein, Austria, Sept.10-15, 2015.

[13] T. Gineste, M. Belhaj, G. Teysedre and J. Puech, "Investigation of the electron emission properties of silver: from exposed to ambient atmosphere $\mathrm{Ag}$ surface to ion-cleaned Ag surface", Appl. Surf. Sci., vol. 395, pp.398-404, Dec. 2015.

[14] C. Inguimbert, M. Belhaj, J. Puech, and J. Pierron. (2014, September) "Monte Carlo simulation of the electron range/energy relationship in the sub$\mathrm{keV}$ energy domain". Presented at MULCOMPIM Conference. [Online]. Available: Doi 10.13140/2.1.2159.4880

[15] F. Salvat, A. Jablonski, and C. J. Powell. "ELSEPA - Dirac partial-wave calculation of elastic scattering of electrons and positrons by atoms, positive ions and molecules", Comp. Phys. Com., vol. 165, n², pp. 157-190, Jan. 2005. [16] D. Edwards, in "Handbooks of Optical Constants", edited by E. D. Palik, Academic, New York, 1985. ISBN: 978-0-12-544415-6, 1997.

[17] J. P. Ganachaud and M. Cailler, "A Monte-Carlo calculation of the secondary electron emission of normal metals", Surf. Sci., vol. 83, n², pp. 498518, May 1979.

[18] H. Shinotsuka, S. Tanuma, C. J. Powell, and D. R. Penn, "Calculations of electron inelastic mean free paths. X. Data for 41 elemental solids over the 50 $\mathrm{eV}$ to $200 \mathrm{keV}$ range with the relativistic full Penn algorithm", Surf. Interface. Anal., vol. 47, $\mathrm{n}^{\circ} 12$, pp. 871-888, Oct. 2015.

[19] C.J. Tung, J.C. Ashley, and R.H. Ritchie, "Electron inelastic mean free paths and energy losses in solids II". Surf. Sci, vol. 81, n², pp. 409-426, Mar.1979.

[20] K. Kumagai, S. Tanuma, and C. J. Powell, "Energy dependence of electron stopping powers in elemental solids over the $100 \mathrm{eV}$ to $30 \mathrm{keV}$ energy range", Nucl. Instrum. Meth. B., vol. 267, n 1, pp. 167-170, Nov. 2009.

[21] N. Cao, B. Da, Y. Ming, S. F. Mao, K. Goto, and Z. J. Ding, "Monte Carlo simulation of full energy spectrum of electrons emitted from silicon in Auger electron spectroscopy", Surf. Interface. Anal., vol. 47, nº 1 , pp. 113-119, Jan. 2015.

[22] I. Bronstein, and B. Fraiman, "Secondary electron emission (in Russian)", Nauka Moskva, 1969.

[23] C. Inguimbert, J.Pierron, M. Belhaj, and J. Puech, "Extrapolated range expression for electrons down to $\sim 10 \mathrm{eV}$ ". Presented at NSREC 2016 Conference (to be published).

[24] O. Kurniawan, and V.K.S. Ong, "Investigation of range-energy relationships for low-energy electron beams in silicon and gallium nitride", Scanning, vol. $29, n^{\circ} 6$, pp. 208-286, Oct. 2007.

[25] A. Wambersie, M. Inokuti, S.M. Seltzer, Journal of the ICRU 7 (2007)

[26] M. H. Bettega, M. A. P. Lima, and L. G. Ferreira, "Electron collisions with CS ${ }_{2}$ ", J. Phys. B. At. Mol. Opt.Phys., vol. 38, pp. 2087-2095, Jun. 2005. 